\title{
Unified Analysis of Generalized Selection Combining over Arbitrary Fading Channels
}

\author{
José Cândido Silveira Santos Filho and Michel Daoud Yacoub
}

\begin{abstract}
We derive general, unified expressions for the probability density function, cumulative distribution function, and mean SNR of generalized selection combining operating over multibranch, arbitrary, correlated, non-identical fading channels. The expressions are written in terms of the joint probability density function of the branch signals and allow for the equalgain and maximal-ratio methods in the combining stage. As an application example, we analyze the case of exponentially correlated Rayleigh channels. The analytical results are fully validated through Monte-Carlo simulations. In passing, new important expressions for the joint distribution and density of the order statistics of arbitrary correlated variates are also derived.
\end{abstract}

Keywords-Arbitrary fading channels, generalized selection combining, order statistics.

\section{INTRODUCTION}

Much attention has been recently given to the generalized selection combining (GSC) scheme. This technique was first proposed in [1], [2] as a suitable tradeoff between performance and complexity and has found applicability in rake receivers for wideband and ultra-wideband communication systems [3]. The GSC chooses the $L$ largest signals out of $M$ available branches and combine them on a equal-gain (EGC) or maximal-ratio (MRC) basis. The respective hybrid schemes are called here selection/equal-gain combining (SEC) and selection/maximal-ratio combining (SMC).

Most published works on GSC are limited to independent diversity channels (see, for instance, [3], [4] and references therein). In practical systems, however, some sort of correlation among the channels often occurs, and the investigation on how this affects the performance is certainly of interest. The SMC performance over identically distributed, identically correlated Nakagami- $m$ channels has been investigated in [5]. In this work, we provide general, unified expressions for the probability density function (PDF), cumulative distribution function (CDF), and mean SNR of SEC and SMC operating over multibranch, arbitrary, correlated, non-identical fading channels. The expressions are written in terms of the joint PDF of the branch signals. To illustrate the usefulness of our general unified approach, sample numerical results are examined for the case of exponentially correlated Rayleigh channels-clearly, our expressions can be applied to any other fading scenario for which the joint envelope PDF is known. Monte-Carlo simulations fully validate the analytical results.

The authors are with the Department of Communications, School of Electrical and Computer Engineering, University of Campinas, PO Box 6101, 13083-852 Campinas, SP, Brazil (Tel: +55 (19) 3788 5106, Fax: +55 (19) 3289 1395, E-mail:[candido,michel]@decom.fee.unicamp.br).
In passing, new important expressions for the joint PDF and CDF of the order statistics of arbitrary correlated variates are also derived.

\section{System ModeL}

Consider $M$ correlated non-identically distributed branch envelopes $R_{1}, \ldots, R_{M}$ following an arbitrary joint PDF $f_{R_{1}, \ldots, R_{M}}(\cdots)$. At any instant, the SEC and SMC schemes choose the $1 \leq L \leq M$ largest branch signals and combine them via EGC and $\mathrm{MRC}$, respectively. Let $O_{1} \geq O_{2} \geq$ $\cdots \geq O_{M}$ denote the order statistics of $R_{1}, \ldots, R_{M}$. Then, the combiner output envelope $R$ can be written as

$$
R= \begin{cases}\sum_{n=1}^{L} O_{n} / \sqrt{L}, & \text { SEC } \\ \sqrt{\sum_{n=1}^{L} O_{n}^{2}}, & \text { SMC }\end{cases}
$$

and $\gamma=R^{2} / 2 N_{0}$ is the output SNR, $N_{0}$ being the noise power in each branch. Next, we derive the PDF $f_{R}(\cdot)$ and CDF $F_{R}(\cdot)$ of $R$ in terms of $f_{R_{1}, \ldots, R_{M}}(\cdots)$. In addition, the mean SNR $\bar{\gamma}=\mathrm{E}\{\gamma\}$ is also attained. (E $\{\cdot\}$ denotes expectation.)

\section{ORDER STATISTICS}

It is clear from (1) that $R$ is a function of $O_{1}, \ldots, O_{L}$. Thus, our first task is to find the joint PDF $f_{O_{1}, \ldots, O_{L}}(\cdots)$. An event-based representation for the corresponding joint CDF $F_{O_{1}, \ldots, O_{L}}(\cdots)$ is given by

$$
\begin{gathered}
F_{O_{1}, \ldots, O_{L}}\left(o_{1}, \ldots, o_{L}\right)=\operatorname{Prob}\left(\bigcup _ { n _ { 1 } = 1 } ^ { M } \left\{R_{n_{1}} \leq o_{1} ; R_{n_{2}} \leq o_{2}\right.\right. \\
\left.\left.\forall n_{2} \neq n_{1} ; \cdots ; R_{n_{L}} \leq o_{L} \forall n_{L} \neq n_{1}, \ldots, n_{L-1}\right\}\right)
\end{gathered}
$$

which can be expressed in terms of $f_{R_{1}, \ldots, R_{M}}(\cdots)$ as

$$
\begin{gathered}
F_{O_{1}, \ldots, O_{L}}\left(o_{1}, \ldots, o_{L}\right)=\sum_{n_{1}=1}^{M} \sum_{\substack{n_{2}=1 \\
n_{2} \neq n_{1}}}^{M} \ldots \sum_{\substack{n_{n_{L-1}=1} \\
n_{L-1} \neq n_{1}, \ldots, n_{L-2}}}^{M} \\
\overbrace{\int_{0}^{O_{L}} \cdots \int_{0}^{o_{L}} \int_{0}^{o_{L}} \cdots \int_{0}^{o_{1}} f_{R_{1}, \ldots, R_{M}}\left(r_{1}, \ldots, r_{M}\right)}^{d r_{n_{1}} \cdots d r_{n_{L-1}} \mathbf{d} \mathbf{r}_{n_{L-1}}, \quad o_{1} \geq \cdots \geq o_{L}}
\end{gathered}
$$

where $\mathbf{d r}_{n_{k}}, 1 \leq k<M$, denotes the $M-k$ differentials present in $d r_{1}, \ldots, d r_{M}$ but not in $d r_{n_{1}}, \ldots, d r_{n_{k}}$. Then, 
differentiating (3), the desired joint PDF is obtained as

$$
\begin{gathered}
f_{O_{1}, \ldots, O_{L}}\left(o_{1}, \ldots, o_{L}\right)=\sum_{n_{1}=1}^{M} \sum_{\substack{n_{2}=1 \\
n_{2} \neq n_{1}}}^{M} \ldots \sum_{\substack{n_{L}=1 \\
n_{L} \neq n_{1}, \ldots, n_{L-1}}}^{M} \\
\overbrace{\int_{0}^{o_{L}} \cdots \int_{0}^{o_{L}}}^{M-L} f_{R_{n_{1}}, \ldots, R_{n_{L}}, \mathbf{R}_{n_{L}}}\left(o_{1}, \ldots, o_{L}, \mathbf{r}_{n_{L}}\right) \mathbf{d r}_{n_{L}}
\end{gathered}
$$

where $\mathbf{R}_{n_{k}}$ and $\mathbf{r}_{n_{k}}, 1 \leq k<M$, denote the $M-k$ branch envelopes not appearing in $R_{n_{1}}, \ldots, R_{n_{k}}$ and their respective sample values. Note, in (4), that $f_{R_{n_{1}}, \ldots, R_{n_{L}}, \mathbf{R}_{n_{L}}}\left(r_{n_{1}}, \ldots, r_{n_{L}}, \mathbf{r}_{n_{L}}\right)=f_{R_{1}, \ldots, R_{M}}\left(r_{1}, \cdots, r_{M}\right)$ $\forall n_{1}, \ldots, n_{L}$. Expressions (3) and (4) are important new generalizations of previous results on order statistics for independent branches (see, for instance, [1, Eqs. (5) and (6)]) and a crucial step to our generalized solutions.

\section{Output Statistics}

Now, the CDF of $R$ can be obtained by integrating (4) over the $L$-dimensional volume defined by

$$
\left\{\begin{array}{l}
o_{1} \geq o_{2} \geq \cdots \geq o_{L} \text { and } \sum_{n=1}^{L} o_{n} / \sqrt{L} \leq r, \quad \text { SEC } \\
o_{1} \geq o_{2} \geq \cdots \geq o_{L} \text { and } \sqrt{\sum_{n=1}^{L} o_{n}^{2}} \leq r, \quad \text { SMC }
\end{array}\right.
$$

This geometrical approach has been used in [4]. Here, we develop a unified treatment for SEC and SMC. From (5), it is not difficult to find the appropriate integration limits for each order statistic. The resulting output $\mathrm{CDF}$ is

$$
\begin{aligned}
& F_{R}(r)=\int_{i_{L}}^{s_{L}} \cdots \int_{i_{1}}^{s_{1}} f_{O_{1}, \ldots, O_{L}}\left(o_{1}, \ldots, o_{L}\right) d o_{1} \cdots d o_{L} \\
& \left(i_{k}, s_{k}\right)=\left\{\begin{array}{l}
\left(o_{k+1}, \frac{\sqrt{L} r-\sum_{n=k+1}^{L} o_{n}}{k}\right), \\
\left(o_{k+1}, \sqrt{\frac{r^{2}-\sum_{n=k+1}^{L} o_{n}^{2}}{k}}\right), k<L, \text { SEC } \\
\left(0, \frac{r}{\sqrt{L}}\right), k=L, \quad \text { SMC }
\end{array}\right.
\end{aligned}
$$

where $f_{O_{1}, \ldots, O_{L}}(\cdots)$ is given in (4). Differentiating (6) with respect to $r$ yields the output PDF

$$
\begin{gathered}
f_{R}(r)=\int_{i_{L}}^{s_{L}} \cdots \int_{i_{2}}^{s_{2}} \dot{s}_{1} f_{O_{1}, \ldots, O_{L}}\left(s_{1}, o_{2}, \ldots, o_{L}\right) d o_{2} \cdots d o_{L} \\
\dot{s}_{1} \triangleq \frac{d s_{1}}{d r}= \begin{cases}\sqrt{L}, \quad \text { SEC } \\
\frac{r}{\sqrt{r^{2}-\sum_{n=2}^{L} o_{n}^{2}}}, & \text { SMC }\end{cases}
\end{gathered}
$$

Note from (4), (6), and (8) that $f_{R}(\cdot)$ and $F_{R}(\cdot)$ are written as sums of finite-range $(M-1)$-fold and $M$-fold integrals of $f_{R_{1}, \ldots, R_{M}}(\cdots)$, respectively. These results are general and can be applied to any fading environment for which $f_{R_{1}, \ldots, R_{M}}(\cdots)$ is known.

Using (8), the mean SNR of GSC in arbitrary fading can be calculated as $\bar{\gamma}=\int_{0}^{\infty} \frac{r^{2}}{2 N_{0}} f_{R}(r) d r$. However, evaluating it numerically renders difficult convergence, due to the infinite upper limit and to the intricacy of $f_{R}(\cdot)$ as shown in (8).

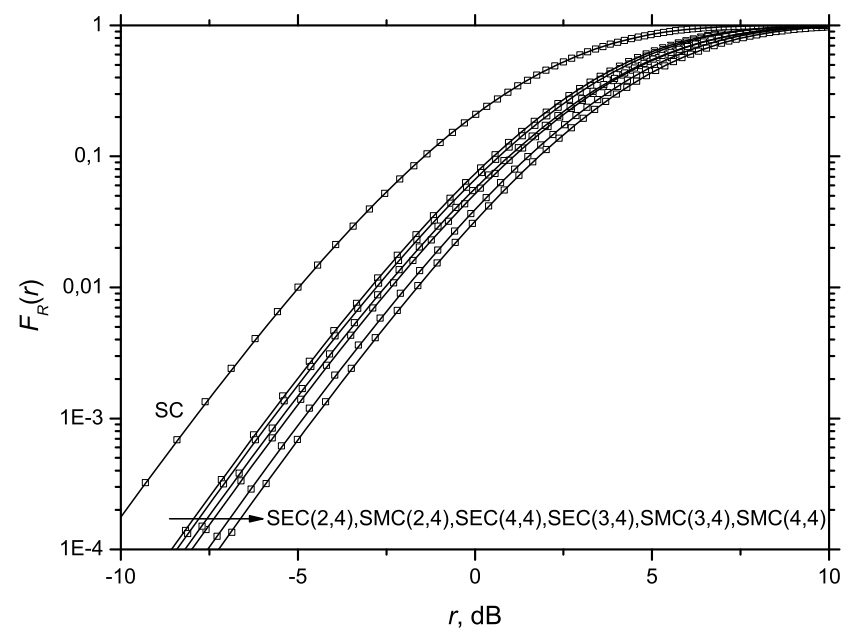

Fig. 1. CDF of SEC and SMC for exponentially correlated Rayleigh branches with $\rho=1 / 2$ (analytical solution: solid; simulation: scatter).

This problem can be circumvented by the change of variables $s=\exp (-r)$, yielding a more efficient integral representation

$$
\bar{\gamma}=\int_{0}^{1} \frac{\ln ^{2} s}{2 N_{0} s} f_{R}(-\ln s) d s
$$

We reinforce that (8) is intended to be replaced into (10), so that the mean SNR of GSC is also written as a finite-range $M$-fold integral of $f_{R_{1}, \ldots, R_{M}}(\cdots)$.

\section{Application Example}

In this section, to illustrate the usefulness of our new general unified expressions, we apply (6) and (10) to obtain the CDF and the mean SNR of SEC and SMC over unitpower exponentially correlated Rayleigh channels, whose joint envelope PDF is given by [6, Eq. (11)]

$$
\begin{gathered}
f_{R_{1}, \ldots, R_{M}}\left(r_{1}, \ldots, r_{M}\right)=\frac{2^{M} r_{M}}{\left(1-\rho^{2}\right)^{M-1}} \\
\exp \left(-\frac{r_{1}^{2}+r_{M}^{2}+\left(1+\rho^{2}\right) \sum_{\substack{n=1 \\
n \neq 1, M}}^{M} r_{n}^{2}}{1-\rho^{2}}\right) \\
\prod_{n=1}^{M-1} r_{n} I_{0}\left(2\left|\frac{\rho}{1-\rho^{2}}\right| r_{n} r_{n+1}\right)
\end{gathered}
$$

where $\rho^{|n-m|}$ is the correlation coefficient between the underlying Gaussian components of the Rayleigh envelopes at the $n$th and $m$ th branches, $n \neq m$. In this case, (6) and (10) can not be solved in closed-form and must be evaluated numerically. For practical values of $M$ (e.g. $M \leq 5$ ), the $M$ fold numerical integrations can be easily attained in standard software packages, although they become intricate as $M$ increases. The resulting curves are shown in Figs. 1 and 2 for $M=4$ and have been computed in Mathematica. In order to validade the new analytical expressions, Monte-Carlo simulation results are also shown in scatter, and an excellent match is verified in all of the cases. 


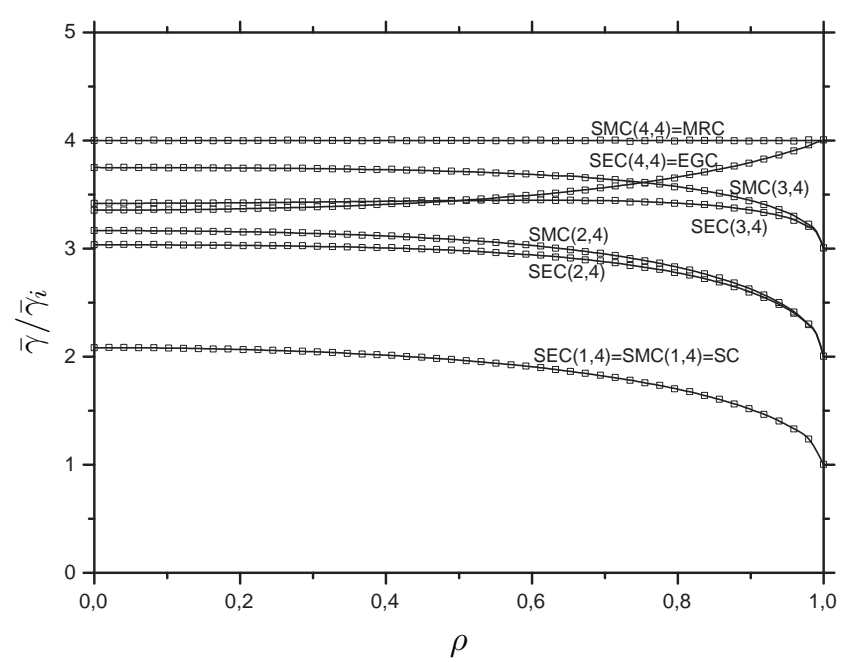

Fig. 2. Mean SNR of SEC and SMC for exponentially correlated Rayleigh branches (analytical solution: solid; simulation: scatter).

In the figures, the GSC schemes are denoted by $\operatorname{SEC}(L, M)$ and $\operatorname{SMC}(L, M)$, and $\bar{\gamma}_{i}$ stands for the mean value of the $\mathrm{SNR} \gamma_{i}$ at each branch. Of course, $\operatorname{SEC}(1, M) \equiv \operatorname{SMC}(1, M)$, $\operatorname{SEC}(M, M)$, and $\operatorname{SMC}(M, M)$ are the well-known selection combining (SC), EGC, and MRC schemes. Note that SMC improves as $L$ increases and always outperforms SEC. Indeed, the performance of SEC approaches that of SMC as $L$ decreases and/or $\rho$ increases. As expected, in Fig. $2, \bar{\gamma} \rightarrow L \bar{\gamma}_{i}$ for both SEC and SMC as $\rho \rightarrow 1$. More interestingly, for SECas opposed to SMC - the increase of $L$ does not necessarily improve the performance. Note, for instance, in Fig. 2 for $\rho \lesssim 1 / 2$ and in Fig. 1 , that $\operatorname{SEC}(3,4)$ outperforms $\operatorname{SEC}(4,4)$. This intriguing behavior of SEC has already been reported for independent branches in [4]: it may be that the inclusion of the signal of one more branch does not overcome the addition of the noise at that branch.

\section{CONCLUSIONS}

In this work, general, unified expressions for the PDF, CDF, and mean SNR of SEC and SMC operating over multibranch, arbitrary, correlated, non-identical fading channels have been derived in terms of the joint PDF of the branch signals. The new expressions apply to any fading scenario for which the joint envelope PDF is known, and their use has been illustrated for exponentially correlated Rayleigh channels. Monte-Carlo simulations fully validated the analytical results. In passing, new important expressions for the joint PDF and CDF of the order statistics of arbitrary correlated variates have been also derived.

\section{REFERENCES}

[1] N. Kong, T. Eng, and L. B. Milstein, "A selection combining scheme for rake receivers," in Proc. IEEE ICUPC, 1995, pp. 426-430.

[2] T. Eng, N. Kong, and L. B. Milstein, "Comparison of diversity combining techniques for Rayleigh-fading channels," IEEE Trans. Commun., pp. 1117-1129, Sept. 1996.
[3] C. Tellambura and A. Annamalai, "Unified performance bounds for generalized selection diversity combining in fading channels," in Proc. IEEE WCNC, vol. 1, Mar. 2003, pp. 138-143.

[4] C. R. C. M. da Silva and M. D. Yacoub, "A generalized solution for diversity combiing techniques in fading channels," IEEE Trans. Microwave Theory Tech., vol. 50, no. 1, pp. 46-50, Jan. 2002.

[5] R. K. Mallik and M. Z. Win, "Analysis of hybrid selection/maximal-ratio combining in correlated Nakagami- $m$ fading," IEEE Trans. Commun., vol. 50, no. 8, pp. 1372-1383, Aug. 2002.

[6] G. K. Karagiannidis, D. A. Zogas, and S. A. Kotsopoulus, "An efficient approach to multivariate Nakagami- $m$ distribution using Green's matrix approximation," IEEE Transactions on Wireless Communications, vol. 2, no. 5, pp. 883-889, Sept. 2003. 